\title{
Canted Antiferromagnetic Phase in a Double Quantum Well in a Tilted Quantizing Magnetic Field
}

\author{
V.S. Khrapai, E. V. Deviatov, A. A. Shashkin, and V. T. Dolgopolov \\ Institute of Solid State Physics, Chernogolovka, Moscow District 142432, Russia \\ F. Hastreiter and A. Wixforth \\ Ludwig-Maximilians-Universität, Geschwister-Scholl-Platz 1, D-80539 München, Germany \\ K. L. Campman and A.C. Gossard \\ Materials Department and Center for Quantized Electronic Structures, University of California, Santa Barbara, California 93106
}

(Received 29 March 1999)

\begin{abstract}
We investigate the double-layer electron system in a parabolic quantum well at filling factor $\nu=2$ in a tilted magnetic field using capacitance spectroscopy. The competition between two ground states is found at the Zeeman splitting appreciably smaller than the symmetric-antisymmetric splitting. Although at the transition point the system breaks up into domains of the two competing states, the activation energy turns out to be finite, signaling the occurrence of a new insulator-insulator quantum phase transition. We interpret the obtained results in terms of a predicted canted antiferromagnetic phase.
\end{abstract}

PACS numbers: $72.20 . \mathrm{My}, 73.40 . \mathrm{Kp}$

Much interest in double-layer systems is aroused by the presence of an additional degree of freedom which is associated with the third dimension. In a double-layer system with symmetric electron density distributions in a normal to the interface magnetic field at filling factor $\nu=$ 2 , the competition of different ground states is expected that is controlled by the relation between the Coulomb interaction energy, the spin splitting, and the symmetricantisymmetric splitting caused by interlayer tunneling. In the simplest single-particle picture, each Landau level has four sublevels originating from the spin and subband splittings. With increasing spin splitting a transition should occur from a spin unpolarized ground state with antiparallel spin orientations of occupied sublevels to a ferromagnetic one with parallel spins when the Zeeman energy $\mu g B$ is equal to the symmetric-antisymmetric splitting $\Delta_{\mathrm{SAS}}$. Experimentally, however, the clear transition was observed at the Zeeman energy significantly smaller than $\Delta_{\text {SAS }}$ $[1,2]$, which points out the importance of many-body effects for the transition. Recent theoretical considerations [3-8] have revealed the crucial role of electronelectron interaction for the spin structure of double-layer electron systems. For the symmetric bilayer electron system in a potential well that is stable to symmetry breaking (so-called easy plane two-dimensional ferromagnet [9]), in addition to the consistent with found in experiment $[1,2]$ shift of the phase transition point to smaller magnetic fields, a new so-called canted antiferromagnetic phase occurs between a spin unpolarized and a ferromagnetic state [3-7]. It is shown that, due to Coulomb repulsion of electrons, mixing the symmetric and antisymmetric states with opposite spin directions forms a new ground state which is a two-particle spin singlet. The transition point between this spin singlet state and the ferromagnetic state in which the spins in both layers point in the direction of the applied magnetic field is defined by the relation

$$
\mu g B \approx \frac{\Delta_{\mathrm{SAS}}^{2}}{E_{c}}
$$

with $E_{c}$ denoting the Coulomb energy. Because normally $E_{c}>\Delta_{\text {SAS }}$, the transition is expected at $\mu g B<\Delta_{\text {SAS. }}$. Near the transition the intralayer exchange interaction connects both lowest states of the electron system and gives rise to the intermediate canted antiferromagnetic phase that is characterized by interlayer antiferromagnetic spin correlations in the two-dimensional plane and is related to the zero-energy spin excitation mode [3-7]. The appearance of this phase signifies a new class of quantum phase transitions between insulators with different spin structures.

The idea of a novel phase has been supported by the recent experiments on inelastic light scattering in which transitions were observed near $\nu=2$ between the ground states of a bilayer system with different spin structures $[10,11]$. However, these states, thus far, have not been studied by transport measurement methods.

The existence of a canted antiferromagnetic phase is predicted for a double layer with asymmetric electron density distributions as well; moreover, external bias allows a continuous tuning of the $\nu=2$ state within a single gated sample [7]. As a result, in the general case, one has two transitions [11] with increasing bilayer asymmetry: ferromagnetic-canted antiferromagnetic-spin unpolarized phase. For a sufficiently large ratio $\Delta_{\mathrm{SAS}} / \mu g B$, the canted antiferromagnetic phase becomes the ground state at the symmetry/balance point, and the first transition disappears. A further increase of the ratio leads to the disappearance of the second transition. In a disordered system, the interval between two transition points is 
expected to have intrinsic structure and can include different spin Bose glass phases [6,7].

Here, we employ a capacitance spectroscopy technique to study the phase transition in the double-layer electron system in a parabolic quantum well at filling factor $\nu=2$ at a tilted magnetic field. The scenario of the observed transition gives strong evidence for a new insulatorinsulator quantum phase transition and supports the formation of the recently predicted canted antiferromagnetic phase, although for tilted magnetic fields a rigorous theory is not yet available.

The samples are grown by molecular beam epitaxy on semi-insulating GaAs substrate. The active layers form a $760 \AA$ wide parabolic well. In the center of the well a three monolayer thick $\mathrm{Al}_{x} \mathrm{Ga}_{1-x} \mathrm{As}(x=0.3)$ sheet is grown which serves as a tunnel barrier between both parts on either side. The symmetrically doped well is capped by $600 \AA \mathrm{AlGaAs}$ and $40 \AA \mathrm{GaAs}$ layers. The sample has Ohmic contacts (each of them is connected to both electron systems in two parts of the well) and two gates on the crystal surface with areas $120 \times 120$ and $220 \times 120 \mu \mathrm{m}^{2}$. The gate electrode enables us both to tune the carrier density in the well, which is equal to $4.2 \times 10^{11} \mathrm{~cm}^{-2}$ at zero gate bias, and measure the capacitance between the gate and the well. For capacitance measurements we apply an ac voltage $V_{\text {ac }}=2.4 \mathrm{mV}$ at frequencies $f$ in the range $3-600 \mathrm{~Hz}$ between the well and the gate and measure both current components as a function of gate bias $V_{g}$ in the temperature interval between $30 \mathrm{mK}$ and $1.2 \mathrm{~K}$ at magnetic fields of up to $14 \mathrm{~T}$.

Our measurements are similar to magnetotransport measurements in Corbino geometry: when disturbed the sample edge becomes equipotential within the edge magnetoplasmon round-trip time $\sim L / \sigma_{x y}$, which is normally much shorter than the time of charge redistribution normal to the edge $\sim C_{0} L^{2} / \sigma_{x x}$, where $\sigma_{x x}$ and $\sigma_{x y}$ are the dissipative and Hall conductivity, $C_{0}$ is the capacitance per unit area between gate and quantum well, and $L$ is the characteristic sample dimension. At low frequencies $f \ll \sigma_{x x} / C_{0} L^{2}$, the imaginary current component reflects the thermodynamic density of states in a double-layer system. In this limit, the, e.g., $\nu=2$ imaginary current component minimum is accompanied by a peak in the active current component which is proportional to $\left(f C_{0}\right)^{2} \sigma_{x x}^{-1}$ and is used for measurements of the temperature dependence of $\sigma_{x x}$. At high frequencies the minimum in the imaginary current component should deepen as caused by in-plane transport so that both current components tend to zero.

The positions of the $\nu=2$ imaginary current component minimum (or active current component maximum) in the $\left(B_{\perp}, V_{g}\right)$ plane are shown in Fig. 1 alongside with those at filling factors $\nu=1,3,4$ for a normal and tilted magnetic fields. In the normal magnetic field, at the gate voltages $V_{t h 1}<V_{g}<V_{t h 2}$, at which one subband is filled with electrons in the back part of the well with respect
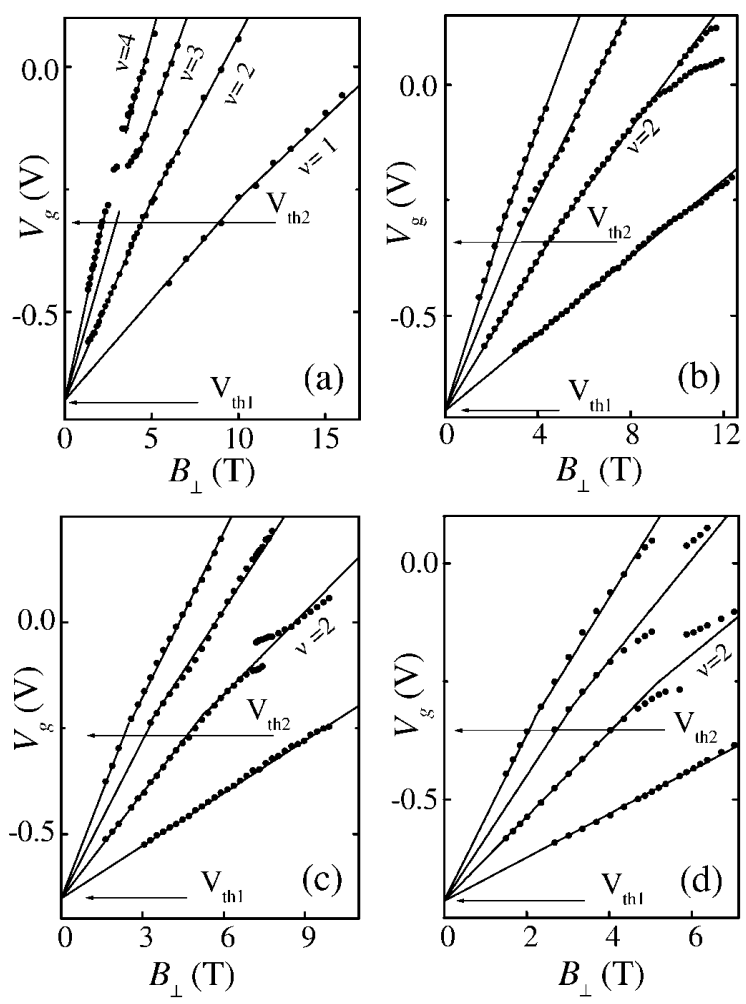

FIG. 1. Positions of the magnetocapacitance minima, or of the active current component maxima, at a temperature of $30 \mathrm{mK}$ for different tilt angles: (a) $\Theta=0^{\circ}$, (b) $\Theta=30^{\circ}$, (c) $\Theta=45^{\circ}$, and $(d) \Theta=60^{\circ}$.

to the gate, the experimental points are placed along a straight line with a slope defined by capacitance between the gate and the bottom electron layer (Fig. 1a). Above $V_{t h 2}$, where a second subband collects electrons in the front part of the well, a minimum in the imaginary current component at integer $\nu$ corresponds to a gap in the spectrum of the bilayer electron system, and the slope is inversely proportional to the capacitance between the gate and the top electron layer [12]. At a tilt angle of $30^{\circ}$, a splitting of the line indicating the position of the $\nu=2$ minimum is observed close to the balance point (Fig. 1b). Knowing $\Delta_{\mathrm{SAS}}=1.3 \mathrm{meV}$ from far infrared measurements and model calculations [13], we can estimate from Eq. (1) the Coulomb energy $E_{c} \approx 6 \mathrm{meV}$ at the transition. This value is smaller than the energy $e^{2} / \varepsilon l=15 \mathrm{meV}$ (where $l$ is the magnetic length) because of finite extension of the electron wave functions in the $z$ direction. As seen from Figs. 1c and $1 \mathrm{~d}$, with increasing tilt angle $\Theta$ the center of the splitting moves towards more negative gate voltages. It is important that the occurrence of two distinct minima at fixed magnetic field points to the competition between the two ground states of our bilayer system.

Figure 2 represents the behavior of the activation energy along the $\nu=2$ line in Fig. 1 at different tilt angles. For $\Theta=0^{\circ}$ at $V_{g}>V_{t h 2}$, the activation energy passes through a maximum and then monotonously decreases with increasing magnetic field. At a tilted magnetic field, there 


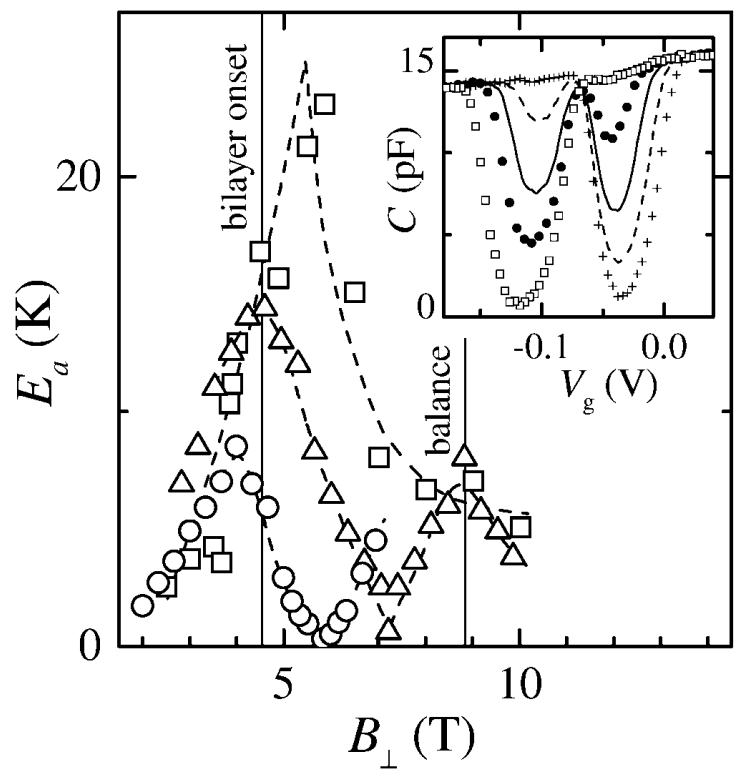

FIG. 2. Change of the activation energy at $\nu=2$ with magnetic field for $\Theta=0^{\circ}$ (squares), $\Theta=45^{\circ}$ (triangles), and $\Theta=$ $60^{\circ}$ (circles). The dashed lines are guides to the eye. The data at $\Theta=30^{\circ}$ are not displayed to avoid overcomplicating the figure. The inset shows the gate voltage dependence of the imaginary current component for $\Theta=45^{\circ}$ at $f=23 \mathrm{~Hz}$ at $T=30 \mathrm{mK}$ for different magnetic fields: 10.03 (squares), 10.19 (dots), 10.28 (solid line), 10.36 (dashed line), and 10.53 T (crosses).

emerges a deep minimum of the activation energy at the field corresponding to the splitting point. We find that for all tilt angles the minimum activation energy is finite (Fig. 2).

A set of the experimental traces near the splitting point for $\Theta=45^{\circ}$ is displayed in the inset of Fig. 2. An interplay is seen of two deep minima in the magnetocapacitance at filling factors slightly above and slightly below $\nu=2$, respectively, which correspond to maxima of the activation energy with a minimum in between at exactly $\nu=2$. At the splitting point both magnetocapacitance minima are observable simultaneously with roughly equal amplitudes.

It is interesting to compare our experimental findings with results of Ref. [2] where a double-layer system with higher mobility and smaller $\Delta_{\text {SAS }}$ was investigated at $\nu=$ 2 at a normal magnetic field. In both experiments a change of the ground state at the balance point is reached by varying a tuning parameter: a total electron density in Ref. [2] and a tilt angle in our case. In addition, we observe the coexistence of two ground states near the transition point and find a finite value of the activation energy at the transition, i.e., it is insulator-insulator transition.

To verify that our experimental results are beyond the single-particle model, we compare them with the singleparticle spectrum in a tilted magnetic field calculated in self-consistent Hartree approximation (details of calculation will be published elsewhere). In the calculation we do not take into account the spin splitting (supposing small $g$ factor) as well as the exchange energy.
For this simplest case the calculated gap for filling factor $\nu=2$ also exhibits a minimum (Fig. 3). It is quite easy to understand the physical origin of the minimum: on one hand, a parallel component of the magnetic field leads to increasing the subband energies because of narrowing the electron density distribution in the $z$ direction. As a result, $\Delta_{\text {SAS }}$ increases a little. On the other hand, to form a gap in the spectrum the tunneling between the layers should occur with conservation of the in-plane momentum. Therefore, it is accompanied with a shift of the center of the in-plane wave function by an amount $d_{0} \tan \Theta$ (where $d_{0}$ is the distance between the centers of mass for electron density distributions in two lowest subbands) which enhances with both deviation from the balance point due to the increase of $d_{0}$ and tilting the magnetic field. The increase of the effective tunneling distance $\sqrt{d^{2}+d_{0}^{2} \tan ^{2} \Theta}$ (where $d$ is the tunnel barrier width) results in decreasing the subband spacing. The combination of these contributions, taking into account the known behavior of the $\nu=2$ gap in the normal magnetic field (see Fig. 3), gives rise to the nonmonotonous dependences of the gap at tilted magnetic fields. From the calculation it follows that the position of the minimum shifts to lower magnetic fields $B_{\perp}$ with increasing tilt angle, which is consistent with our experimental finding. Nevertheless, the measured minimum activation energy is far smaller than the calculated half gap.

In principle, lower values of the measured activation energy might be explained in the single-particle picture assuming that the quantum level width is finite. However, we reject such an explanation for the following reasons: first, at $\Theta=0^{\circ}$ at balance we do observe an activation energy very close to half of $\Delta_{\text {SAS }}$ and practically at the same magnetic field where a deep minimum in the activation energy is observed at $\Theta=30^{\circ}$; second, when sweeping the gate

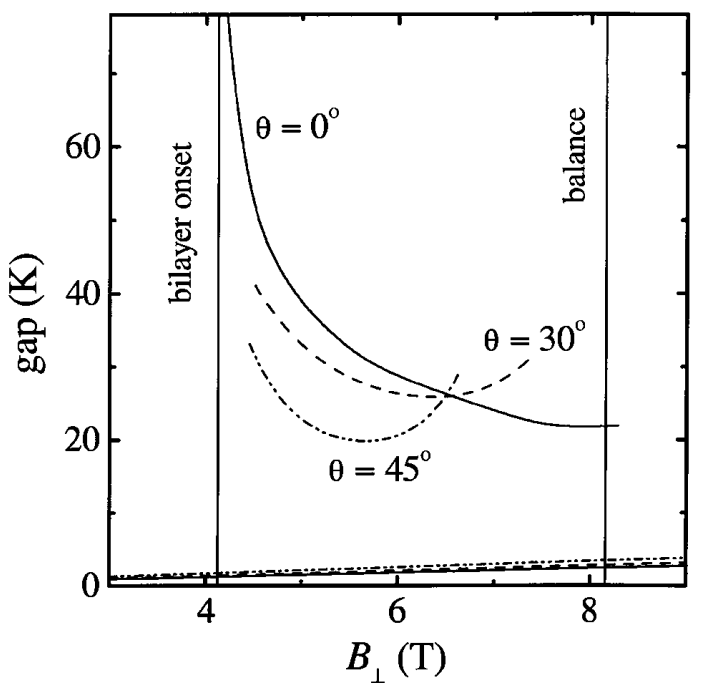

FIG. 3. Results of the $\nu=2$ gap calculation in self-consistent Hartree approximation for $\Theta=0^{\circ}, \Theta=30^{\circ}$, and $\Theta=45^{\circ}$. Also shown is the corresponding Zeeman splitting. 
voltage through the splitting point the activation energy shows two maxima with a nonzero minimum in between at $\nu=2$, which indicates the coexistence of two ground states in the form of domains at the critical point and is in contradiction to the single-particle picture.

To apply the line of reasoning developed in a many-body lattice model [6] to the case of a tilted magnetic field, one has to introduce two significant changes: (i) as one rung, one should consider two sites shifted on distance $d_{0} \tan \Theta$; (ii) the value of $\Delta_{\mathrm{SAS}}$ should be replaced by the subband spacing as determined from self-consistent Hartree approximation. Then, the conclusion of Ref. [6] about the existence of an intermediate canted antiferromagnetic phase is expected to remain valid. We note that the theory [4-7] deals with a change of the ground state while experimentally we measure the energy of a charge excitation with $k=\infty$ [14]. Therefore, the comparison of theory with experiment is straightforward only in the single-particle picture where the nearest excited state is identical with the competing ground state. According to Ref. [5], in the many-body problem peculiarities of the activation energy are expected near the phase transition.

In our opinion, the observed deep minimum in activation energy at tilted magnetic fields is a manifestation of transition from a spin unpolarized state to a canted antiferromagnetic phase: at gate voltages right above $V_{t h 2}$ the spin unpolarized state only can be realized for filling factor $\nu=2$. The ferromagnetic and canted antiferromagnetic phase should then be considered as possible states near the balance point. At the transition point a finite activation energy is found, which is not the case for the direct transition from the spin unpolarized to the ferromagnetic state [5]. Hence, the transition scenario forces us to recognize the phase around the balance point as a canted antiferromagnetic phase in a disordered sample, or, as shown in Refs. [6,7], a Bose glass of the singlet bosons.

In summary, we have studied the double-layer electron system in a parabolic quantum well at $\nu=2$ at tilted magnetic fields using capacitance spectroscopy. We observe a change of the ground state of the system at the Zeeman splitting far smaller than $\Delta_{\text {SAS. }}$. At the transition point, the activation energy is found to be finite although the ground state is composed of domains of two competing states. Our data correspond well to insulator-insulator quantum phase transition from a spin unpolarized to canted antiferromagnetic state in a disordered system [6].
We are thankful to V. Pellegrini for valuable discussions. This work was supported in part by Deutsche Forschungsgemeinschaft, AFOSR under Grant No. F49620-94-1-0158, the Russian Foundation for Basic Research under Grants No. 97-02-16829 and No. 98-02-16632, and the Programme "Nanostructures" from the Russian Ministry of Sciences under Grant No. 97-1024. The Munich-Santa Barbara collaboration has also been supported by a joint NSF-European Grant and the Max-Planck research award.

[1] G. S. Boebinger, H.W. Jiang, L. N. Pfeiffer, and K.W. West, Phys. Rev. Lett. 64, 1793 (1990).

[2] A. Sawada, Z.F. Ezawa, H. Ohno, Y. Horikoshi, Y. Ohno, S. Kishimoto, F. Matsukura, M. Yasumoto, and A. Urayama, Phys. Rev. Lett. 80, 4534 (1998).

[3] L. Zheng, R. J. Radtke, and S. Das Sarma, Phys. Rev. Lett. 78, 2453 (1997).

[4] S. Das Sarma, S. Sachdev, and L. Zheng, Phys. Rev. Lett. 79, 917 (1997).

[5] S. Das Sarma, S. Sachdev, and L. Zheng, Phys. Rev. B 58, 4672 (1998).

[6] E. Demler and S. Das Sarma, Phys. Rev. Lett. 82, 3895 (1999).

[7] L. Brey, E. Demler, and S. Das Sarma, Phys. Rev. Lett. 83, 168 (1999).

[8] T. Jungwirth, S. P. Shukla, L. Smrcka, M. Shayegan, and A. H. MacDonald, Phys. Rev. Lett. 81, 2328 (1998).

[9] A. H. MacDonald, P. M. Platzman, and G.S. Boebinger, Phys. Rev. Lett. 65, 775 (1990).

[10] V. Pellegrini, A. Pinczuk, B. S. Dennis, A. S. Plaut, L. N. Pfeiffer, and K. W. West, Phys. Rev. Lett. 78, 310 (1997).

[11] V. Pellegrini, A. Pinczuk, B. S. Dennis, A. S. Plaut, L. N. Pfeiffer, and K. W. West, Science 281, 799 (1998).

[12] V.T. Dolgopolov, A. A. Shashkin, E. V. Deviatov, F. Hastreiter, M. Hartung, A. Wixforth, K. L. Campman, and A. C. Gossard, Phys. Rev. B 59, 13235 (1999).

[13] M. Hartung, A. Wixforth, K. L. Campman, and A. C. Gossard, Solid State Electron. 40, 113 (1996); G. Salis, B. Graf, K. Ensslin, K. Campman, K. Maranowski, and A. C. Gossard, Phys. Rev. Lett. 79, 5106 (1997).

[14] Both the shift of the ground state energy and the exchange-enhanced $g$ factor are caused by electron-electron interaction. Nevertheless, these effects are quite different because the former corresponds to $k=0$ while the latter describes excitations with $k \gg 1 / l$. This difference was not clearly expressed in Ref. [12]. 